\title{
Guest Editorial Column
}

\section{Mqhele Enock-Hershal Dlodlo $\cdot$ Neeli R. Prasad ${ }^{2}$ Ivan Seskar ${ }^{3} \cdot$ Thomas Magedanz $^{4}$. Albena Mihovska ${ }^{5}$}

Published online: 30 April 2021

(c) The Author(s), under exclusive licence to Springer Science+Business Media, LLC, part of Springer Nature 2021

The thirteen articles in the Special Issue on the theme "Towards a virtualized communications world-Challenges and Opportunities" present a diversity of applications enabled by $5 \mathrm{G}$ and beyond. In addition, some of them present results of performance improvements and greater online security. The focus on humans opens doors to groundbreaking business modelling innovations.

The first three studies focus on functional and personalised support services to the user. For instance, in the first study, senior citizens can now benefit from a context-aware smart care applications to select food from a personalised menu, given their clinical requirements. Digital literacy in the second study extends access to previously marginalised young persons by creating a personalised smart learning environment for the digitally transformed world. In the third study, the authors improve on problem-based learning by introducing a blended learning instruction platform.

The fourth study exploits $5 \mathrm{G}$ cloudlets to enhance driver assistance in vehicular communications to alert drivers to impending hazardous conditions arising from behaviours of other drivers. In a game of football, the fifth study offers an improvement on the then existing expert systems eliminating the twin problems of occlusion and afterimage and thus correcting for possible errors of judgment by referees and linesmen.

Mqhele Enock-Hershal Dlodlo

mqhele.dlodlo@uct.ac.za

Neeli R. Prasad

neeliprasad@gmail.com

Ivan Seskar

seskar@winlab.rutgers.edu

Thomas Magedanz

thomas.magedanz@fokus.fraunhofer.de

Albena Mihovska

amihovska@btech.au.dk

1 University of Cape Town, Cape Town, South Africa

2 ITU To CTO, Smartavatar B.V., Delft, Netherlands

3 Rutgers University, New Brunswick, USA

4 Fokus Fraunhofer Institute, Berlin, Germany

5 Aarhus Universit, Åarhus, Denmark 
The sixth up to the eleventh studies dwell on performance improvements to systems found in 5G and beyond. Study 6 presents results of the performance analysis of a stablematching algorithm with cross-layer optimisation but with imperfect channel-state information. The authors of the seventh study realise energy savings and reliability in WSNs using an advanced data transfer scheme called Adaptive Sectoring Scheme for Reliability (ASSR). Study 8 presents results for Advanced Shifted 2-N-PSK modulation for PHY security. Unified Wireless Access for Smart Cities using large-scale, complex, intelligent Cyber-Physical Systems for humans and things is the subject of the ninth study. The tenth article shows good results from early tests on security threats to Human Bond Communication Systems. Study 11 improves on frontal face recognition in Personal ID systems using a 2D pose-invariant face recognition algorithm.

Using the BIOGAS BMES as a testbed, the twelfth study analyses human behaviour with a novel Business Model (BM) that yielded high speed and a good Return on Investment. The thirteenth study proposes an attribute to determine a student's competency by modifying the "Objective Distance" from the initial status towards a satisfied competency level and the results showed the Modified Distance approach to be an improvement on the original.

Publisher's Note Springer Nature remains neutral with regard to jurisdictional claims in published maps and institutional affiliations.

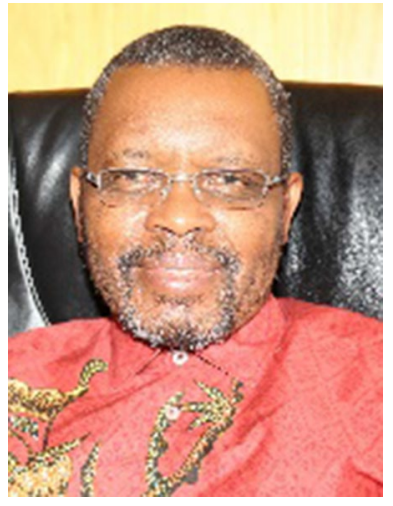

Mqhele Enock-Hershal Dlodlo (FZwelE, MIEEE, Pr.Eng. (ECZ), FZAS) is the Vice-Chancellor of the National University of Science and Technology. At the University of Cape Town, Rondebosch, South Africa, he is an Emeritus Associate Professor in telecommunications in the Electrical Engineering Department. His research interests are in wireless communication systems and applications. An emeritus member of the Communications Research Group (CRG) in the Telkom Centre of Excellence $(\mathrm{CoE})$ for Broadband Networks and Applications he continues to co-supervise PhD students. The research has led to over a hundred international refereed publications, eight Ph.D. theses, numerous master's dissertations and an international IP registration. In the time at UCT, Prof. Dlodlo served as Assistant Dean for Internationalization and Programme Convener for the Master of Engineering (Telecommunications) degree programme. He has also designed curricula, taught, consulted, researched, headed departments and faculties at NUST as well as the Bulawayo Polytechnic over twenty-one years. In 2003 he became a Fulbright Senior African Scholar at Virginia Tech in America, studying Learning and Information Technology Initiatives for academic leadership in research, learning, and fundraising. Home pages: The Vice-Chancellor Prof M E Dlodlo (nust.ac.zw); Emeritus Associate Professor Mqhele Dlodlo I Department of Electrical Engineering (uct.ac.za). 


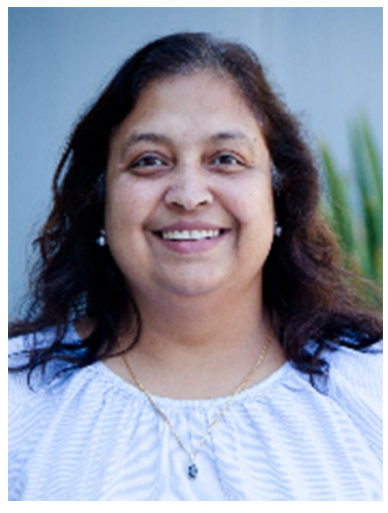

Dr. Ir. Neeli R. Prasad, Professor Department of Business Development and Technology (BTech), Aarhus University, Denmark, Chief Technology Officer of SmartAvatar B.V. and VehicleAvatar Inc., IEEE VTS Board of Governor Elected Member and Vice President Membership Development. Neeli is a cybersecurity, networking and Internet of Things (IoT) strategist. Throughout she has been driving business and technology innovation, from incubation to prototyping to validation and is currently an entrepreneur in Silicon Valley. She has made her way up the waves of secure communication technology by contributing to the most ground-breaking and commercial inventions. She has general management, leadership and technology skills, having worked for service providers and technology companies in various key leadership roles. She is the advisory board member for the European Commission H2020 projects. She is also a vice chair and patronage chair of IEEE Communication Society Globecom/ICC Management and Strategy Committee (COMSOC GIMS) and Chair of the Marketing, Strategy and IEEE Staff Liaison Group. She is Director of CGC, USA and was assistant head of department and Professor, Electrical and Computer Engineering at International Technological University (ITU), USA. Dr. Prasad has led global teams of researchers across multiple technical areas and projects in Japan, India, throughout Europe and USA. She has been involved in numerous research and development projects. She also led multiple EU projects such as Magnet, Magnet II, CRUISE, LIFE 2.0, ASPIRE, BeTaaS, Futon, etc. as project coordinator and PI as research director of CTIF and Associate Professor, Aalborg University, Denmark. She has played key roles from concept to implementation to standardization. Her strong commitment to operational excellence, innovative approach to business and technological problems and aptitude for partnering cross-functionally across the industry have reshaped and elevated her role as project coordinator making her a preferred partner in multinational and European Commission project consortiums. She has 4 books on IoT and WiFi, many book chapters, peer-reviewed international journal papers and over 200 international conference papers. She was IEEE Communication Society distinguished lecturer. Dr. Prasad received her Master's degree in electrical and electronics engineering from Netherland's renowned Delft University of Technology, with a focus on personal mobile and radar communications. She was awarded her Ph.D. degree from Università di Roma "Tor Vergata", Italy, on Adaptive Security for Wireless Heterogeneous Networks. My website: http://neeliprasad.com.

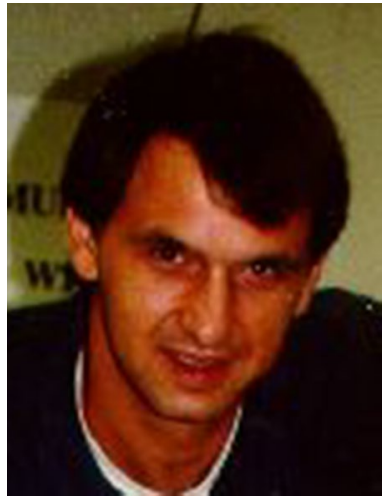

Ivan Seskar is Associate Director at WINLAB, Rutgers University responsible for experimental systems and prototyping projects. He was one of the co-PIs and a lead project engineer for ORBIT, with responsibility for development, integration and deployment of the radio-grid emulator system for which the team received 2008 NSF Alexander Schwarzkopf Prize for Technological Innovation. He is currently the PI for the NSF funded GENI-wide Open LTE/WiMAX deployment, PI for the "meso-scale" Open-Flow campus deployment and PI for the Cloud-RAN "CloudLab at RU" at Rutgers University. His technical interests include experimental protocol evaluation, radio technology, software defined and cognitive radios, vehicular networking and wireless systems in general. Ivan is a Senior Member of the IEEE, member of ACM and co-founder and CTO of Upside Wireless Inc. Ivan Seskar's Home Page: http://www.winlab.rutgers.edu/ seskar/. 


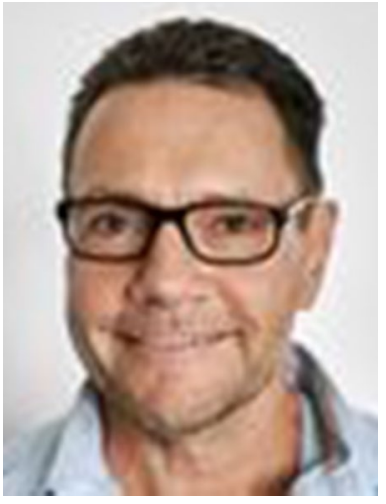

Thomas Magedanz [SM] (magedanz@ieee.org, Thomas.magedanz@ fokus.fraunhofer.de) is a full professor on the electrical engineering and computer sciences faculty at the Technical University of Berlin, Germany, leading the chair for Next Generation Networks. In addition, he is the director of the "Software-based Networks" division of the Fraunhofer Institute FOKUS. In 2006, he was named an Extraordinary Professor in the Department of Electrical Engineering of the University of Cape Town, South Africa. Since 2007, he has also been a visiting professor in the Department of Mathematics, Physics and Computing at the Waterford Institute of Technology in Ireland. For more than 20 years he has been working in the convergence field of fixed and mobile telecommunications, the Internet, and information technologies, which resulted in many international $R \& D$ projects centered around next generation service delivery platforms prototyped in a set of globally recognized open technology testbeds. In 2007, he joined the European FIRE (Future Internet Research and Experimentation) Expert Group. In the course of his research activities, he has published more than 250 technical papers/articles. In addition, he is a senior member of the IEEE, and serves on the editorial board of several journals. He received his diploma and his $\mathrm{Ph} . \mathrm{D}$. in computer sciences from the Technical University of Berlin, Germany, in 1988 and 1993, respectively. In 2000 he finished his postdoctoral lecture qualification in applied computer sciences at the Technical University of Berlin, Germany. (Based on document published on 19 October 2016). Thomas Magdanz's Home Page: https://www.fokus. fraunhofer.de/usr/magedanz .

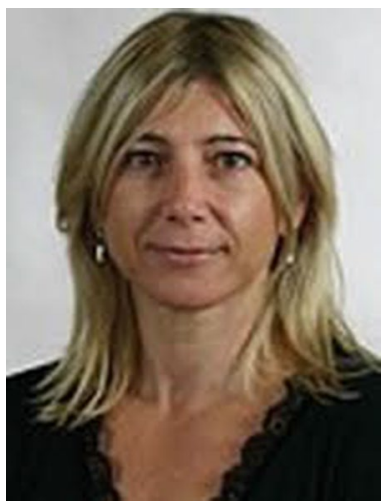

Albena Mihovska is an Associate Professor at Aarhus University, Department of Business Development and Technologies, where she is with the CTIF Global Capsule and MBIT research group. Her main activities relate to research in the area of smart dense connectivity and related applications; $5 \mathrm{G}$ ultradense access networks, Internet of Things technologies for healthcare and smart grid, and most recently, holographic communications. In addition, she focuses on aspects of digital innovations and their impact on business. She was the leader of the local research team within the FP6 EU project WINNER that had a total funding for Aalborg University of 535.000,00 EUR and brought together the most powerful industrial and academic international players at the time in a joint effort to design a new wireless generation air interface, which laid the foundations for the LTE and LTE-A standards. During 1999-2001, she was appointed to the EU-funded project under FP4 ACTS, as part of the technical team. During 2001-2005, she was the Technical Manager of the EU-funded under FP5 project PRODEMIS, which defined a roadmap for the wireless future up to 2010. During 2004-2009, she was the project coordinator of the EU-funded under FP6 project SIDEMIRROR. All three projects were of the special support type of instrument and had as the main objective to concentrate the European research effort by structuring and integrating the results/achievements of the IST FP4-FP6 projects and disseminating these results through four high quality, thematic book publications, published by Artech House. She has strong collaboration skills and has established a large international research network that supports Ph.D. academic research. She has been performing teaching and supervision at undergraduate, graduate and post-graduate level. This has resulted in more than 30 successfully completed graduate projects and $2 \mathrm{PhD}$ thesis. She has been an active contributor to the ITU-T standardization activities in the area of (1) Internet of Things, E-health, and Next Generation Networks (in the period 20102016), (2) Standards education and most recently, SG13 (Future Networks and (cloud)) and SG20 (IoT, Smart cities, and Communities). 